\title{
OPERATIONS OF DRONES IN CONTROLLED AIRSPACE IN EUROPE
}

\author{
Andrija Vidović ${ }^{1}$, Tomislav Mihetec ${ }^{2}$, Bo Wang ${ }^{3}$, Igor Štimac ${ }^{4}$ \\ ${ }^{1,2}$ University of Zagreb, Faculty of Transport and Traffic Sciences, Department of Air Transport, \\ Vukelićeva 4, 10000 Zagreb, Croatia \\ ${ }^{3}$ Ningbo University of Technology, 201 Fenghua Road, Jiangbei District, Ningbo, Zhejiang, China, \\ 315211 \\ ${ }^{4}$ Zagreb Airport Ltd. Ulica Rudolfa Fizira 1, p.p. 40, HR - 10410 Zagreb, Croatia
}

Received 21 November 2018; accepted 10 January 2019

\begin{abstract}
The paper analyses the possibilities for introducing drones in the controlled airspace. It is, namely, inevitable that in the future, drones will become regular participants of civil aircraft operations, and it is therefore necessary, apart from the existing regulatory framework, to define and improve the standards and regulations in order to perform civil operations of drones in the controlled airspace in a safe manner. The purpose of this research is to present better understanding of the rules, standards and regulations, and to address the adaptation in relation to the current situation of performing operations by drones in controlled airspace. The objective of this research is to identify emerging issues of adaptation of operations for safe introduction of drones in the controlled airspace. With regard to abovementioned, the research question should be the following: is the current ATM environment ready for the introduction of commercial drone operations in the controlled ATC.
\end{abstract}

Keywords: drones, Unmanned Aircraft Systems, Remotely Piloted Aircraft System, international regulations, controlled airspace, Air Traffic Management, Air Traffic Control.

\section{Introduction}

Unmanned aircraft systems (UAS) are an aircraft and its associated elements which are operated with no pilot on board. Remotely piloted aircraft systems (RPAS) are a set of configurable elements consisting of a remotely piloted aircraft, its associated remote pilot station(s), the required command and control links and any other system elements as may be required, at any point during flight operations (EASA, 2015). RPAS are a sub-set of UAS. Unmanned aircraft come in a variety of shapes and sizes, ranging from small handheld types up to large aircraft, potentially a similar size to airliners and, just like manned aircraft, they may be of a fixed wing design, rotary winged, or a combination of both. Unmanned Aircraft may also be referred to as:

- Drones;

- Remotely Piloted Aircraft Systems (RPAS);

- Unmanned Aerial Vehicles (UAV);

- Model Aircraft;

- Radio Controlled Aircraft.

All these terms that are coming from the ICAO circular 328-AN/190 and CAA UK CAP 722 are replaced in the common language by the

${ }^{1}$ Corresponding author: avidovic@fpz.hr 
word drone and this paper will accordingly use drones to speak of UAS and RPAS.

Regardless of the name used, they all share the common characteristic that the person responsible for piloting the aircraft is not onboard it. Drone operations in the present aviation environment raises concerns about safety, security, privacy, data protection, and the environment. The challenge is therefore to develop rules that will satisfactorily address those concerns, allowing at the same time the market for drone services to fully develop.

The safe integration of drones into nonsegregated airspace is a long-term activity with many stakeholders adding their expertise on such diverse topics as licensing and medical qualification of remote pilots, technologies for detect and avoid systems, frequency spectrum, separation standards from other aircraft and development of a robust regulatory framework (ICAO, 2015).

The market development of civil drones has the potential to foster jobs and growth in Europe and in the rest of the world. At the same time, drones also raise concerns about safety, security or privacy. That is why Europe needs rules that strikes a balance between promoting drone services and protecting the high levels of safety, security or privacy that European citizens are enjoying. The European aviation community gathered in Riga in March 2015 and established basic principles to guide the regulatory framework in Europe (EC, 2015). The drones need to be treated as new types of aircraft with proportionate rules based on the risk of each operation. EU rules for the safe provision of drone services need to be developed now and technologies and standards need to be developed for the full integration of drones in the European airspace. Public acceptance is key to the growth of drone services and the operator of a drone is responsible for its use. In addition, EUROCONTROL in 2017 hosted a RPAS workshop aiming to create a common picture of the current European integration status of RPAS operations below $500 \mathrm{ft}$ (EUROCONTROL, 2017).

Introduction of commercial drone operations in the existing ATM environment is still not mature enough to mitigate all the risks that are accompanied within this process. The maturity is defined by the level of existing regulation, manufacturers needs to adapt, technology development and influence on society. The evolution and the scale of introduction of commercial drones in the controlled ATC airspace will indicate even greater number of issues that are still not visible.

Therefore, this paper investigates the current commercial use of drones on major business areas and its influence on current ATM operating environment. It should be noted down that ATM as a business area is heavily regulated environment, on both international, regional and local level, and as such, any introduction of "something new" in the system always represents a challenge for overall aviation community. It is more than obvious that introduction of drones in the controlled ATC airspace should undertake risk-based approach with great caution.

The methodology used for this research contains the analysis of current international, regional and national regulatory requirements and future evolution of the regulatory framework. Commercial drone data collected for this paper is based on current up-to date market research data bases, while the ATM data was collected from ICAO, EASA, EUROCONTROL, national CAA and ANSP. In addition, extensive drone market research has been conducted, whereas 
the emphasis has been put on the European market and drone integration roadmap. The assessment of commercial drone introduction in controlled ATC airspace was performed in cooperation with air traffic controllers that have extensive knowledge of ATM environment and that have had an opportunity to coordinate the ATC operations during the drone activities in controlled airspace. Interviews with air traffic controllers were performed and based on the interview outcomes, major issues related to the research problem have been identified. Since air navigation service provider safety-assessments of drone operations in controlled ATC airspace were not disclosed to us, we were not able to investigate even-further all the issues and acceptable levels of safety for such operations.

The expected research results in this paper represent the assessment of the existing state of the drone system for civil purposes in the world and identification of possible issues for the introduction of drones in controlled ATC airspace.

\section{Holistic View on Drone Application in Europe}

In order for drones to be able to use their own full potential, it should be able to fly as

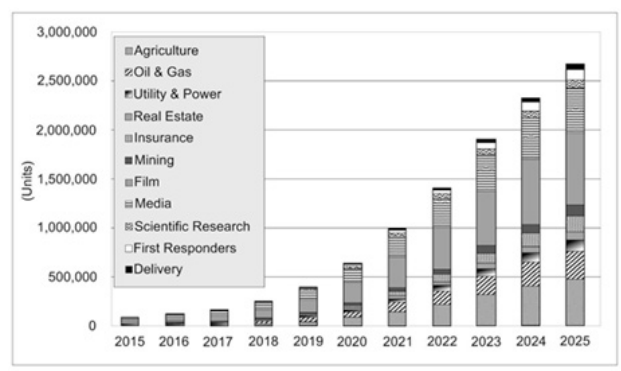

1.a) normal air traffic, and to be integrated in the airspace used by civilian users. EU member countries have started to introduce operations of drones into the controlled airspace with the goal of exploiting these on the new markets; such as monitoring and recording of the infrastructure. It is difficult to predict the full potential of the drone market. According to the sources from the industry, the total budget forecast for drones in the sense of research and development, procurement (by the military and civilian users) will grow from the current $\$ 5.2$ bn to $\$ 11.6$ bn in 2023 (Teal Group Corporation, 2014).

The figure below (1.a) shows the consumer UAV worldwide sales increase in next few years reaching 2.7 million units in 2025 (Embedded Vision Summit, 2016). The demand for UAVs, is driven by the global trends of increasing interest for high definition imaging for personal use, recreational activities, and aerial games. Also, huge potential is for UAVs to create value in commercial markets such as film and media, agriculture, oil and gas, and insurance. Figure 1.b presents the forecast whereas global UAVs services revenue will increase from $\$ 170$ million in 2015 to $\$ 8.7$ billion by 2025 .

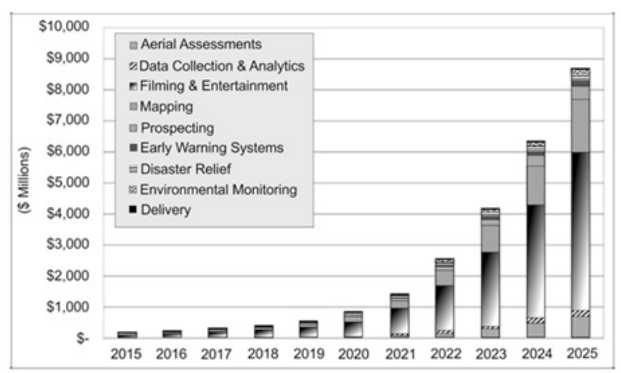

1.b)

Fig. 1.

UAV Market Growth is Expected to be Dramatic in the Coming Years (1.a)); Drone Shipments into Commercial Applications (1.b)). 
Source: (Embedded Vision Summit, 2016)

The European Union has developed a Roadmap that proposes a gradual phase approach to the introduction of drone operations, based on five types of operations and three levels of integration (ERSG, 2013). The RPAS roadmap contains the integration of all the above-mentioned scenarios. The typical flight profiles of drones can comprise a wide range of scenarios, which are categorised in the following types of operations:

(1) Very low level (VLL) operations (alias non-standard VFR or IFR operations) below the typical IFR and VFR altitudes for manned aviation: i.e. below $500 \mathrm{ft}(\sim 150$ m) above ground level; they comprise:

a) Visual line of sight (VLOS) in a range not greater than 500 metres from the remote pilot, in which the remote pilot maintains direct unaided visual contact with the remotely piloted aircraft;

b) Extended Visual Line of Sight (E-VLOS) where, beyond 500 metres, the pilot is supported by one or more observers, in which the drone crew maintains direct unaided visual contact with the remotely piloted aircraft;

c) Beyond VLOS (B-VLOS) where the operations are also below $500 \mathrm{ft}$., but beyond visual line of sight requiring additional technological support.

(2) Drone operations in VFR or IFR, above $500 \mathrm{ft}$ and above minimum flight altitudes; they comprise:

a) IFR (or VFR) operations in radio line-of-sight (RLOS) from drones in non-segregated airspace where manned aviation is present. The key capability to 'detect and avoid' (D\&A) is required in relation to cooperative and non- cooperative nearby traffic (otherwise specific procedures and restrictions would apply);

b) IFR (or VFR) operations beyond radio line-of-sight (BRLOS) operations, when the pilot can no longer be in direct radio contact with the drone and therefore a wider range of communication (COM) services (including via satellite) are necessary. In this case, communications would typically be offered by a COM service provider. BRLOS (SATCOM) operations may apply to long range transport of freight, which is expected to follow flight profiles similar to those used by current manned commercial air transport.

\section{Analysis of International Regulatory Approach on Drones}

The development of drones has opened a promising new chapter in the history of aerospace. Drones offer a wide range of possibilities for the benefit of society, ranging from environmental control and security, as well as a fascinating variety of commercial services. However, the absence of a clear regulatory framework does not currently allow the creation of a truly market for drone services, which limits the potential for jobs and growth creation in this new sector of the economy.

As can be seen in extension of this chapter, there are a number of organizations that deal with issues of standardization of operating procedures and deployment of drones in controlled airspace. In this paper, the focus will be on civil organizations while military organisations, such as NATO and European Defence Agency (EDA) have different approach concerning drones. 


\subsection{ICAO Approach}

In 2011, ICAO issued Circular 328 AN/190, considered as a first attempt to develop an international regulatory framework towards global harmonisation in the regulation of drones. The goal of ICAO in addressing drones is to provide an international regulatory framework through Standards and Recommended Practices (SARPs), with supporting Procedures for Air Navigation Services (PANS) and guidance material, to underpin routine operation of drones throughout the world in a safe, harmonized and seamless manner comparable to that of manned operations (ICAO, 2015).

ICAO RPAS programme covers all relevant subjects as in manned aviation, incorporating drone characteristics: licensing, certification, usage of frequency spectrum, environment, communication / navigation / surveillance, liability concerns, etc.

Recent developments in terms of introducing improvements within the environment for drones have been published within the framework of Global Air Navigation Plan (GANP), called Aviation System Block Upgrades (ASBUs). ASBUs present a package of modules, which would ensure measurable operational improvements and interoperability of systems used in Air Traffic Management. For this paper, most important Performance Improvement Area identified by the ICAO is the "Efficient Flight Path - Through Trajectory-based Operations" that is comprised by three modules. First module Initial Integration of Remotely Piloted Aircraft (RPA) into NonSegregated Airspace foresees the integration of drones into traffic within non-segregated airspace, and fully transparent operation within the airspace. Second module,
Remotely Piloted Aircraft Integration in Traffic present follow-up of previous module and it contains procedures and technology to expand the application of drones and integration in traffic while maintaining the levels of safety. The third module Remotely Piloted Aircraft (RPA) Transparent Management presents the continuation of activities of previous two modules with improvements in the following areas: access to most airspace for select airframes without specific authorization or experimental aircraft waiver, certification procedures for drones, approval procedures for drones, standardized C2 link failure procedures, new special purpose transponder code for C2 link failure, etc.

\subsection{EASA Approach}

The operation of large civilian drones exceeding $150 \mathrm{~kg}$ in European Union Member States is regulated by EU Law and monitored by the European Aviation Service Agency. Smaller drones are largely unregulated at the EU level at this time and are thus regulated by the individual EU Member States. While some individual countries have adopted legislation or implemented temporary provisions on the operation of drones, various regulatory and legislative proposals are currently being considered.

The EASA concept of operations suggests new regulatory concept which is proportionate, progressive, risk based, high level rules complemented by Industry Standards. EASA suggests the implementation of EU rules to cover all drones; implementation depends of the level of risk and that the commercial and non-commercial operations of drones are treated in the same manner (EASA,2015). 
The latest regulatory developments relate to the EASA Opinion 01/2018, that includes a proposal for a new Regulation for UAS operations in 'open' and 'specific' category. According to EASA:

- “open” category is a category of UAS operation that, considering the risks involved, does not require a prior authorisation by the competent authority nor a declaration by the UAS operator before the operation takes place;

- "specific" category is a category of UAS operation that, considering the risks involved, requires an authorisation by the competent authority before the operation takes place, taking into account the mitigation measures identified in an operational risk assessment, except for certain standard scenarios where a declaration by the operator is sufficient or when the operator holds a light UAS operator certificate (LUC) with the appropriate privileges.;

- “certified" category is a category of UA operation that, considering the risks involved, requires the certification of the UAS, a licensed remote pilot and an operator approved by the competent authority, in order to ensure an appropriate level of safety.

Apart from EASA initiatives on safety promotion, the European Commission (i.e. Directorate General (DG) Growth) is developing a multilingual portal, the DronesRules.eu website, under the COSME programme, aiming to support stakeholders' awareness of, and access to, the regulatory framework for UAS operations. The programme covers both recreational and professional UAS operations and focuses on key aspects such as data protection and privacy, safety, as well as liability and insurance.

\subsection{EUROCONTROL Approach}

EUROCONTROL is the leading participant in the work related to the aspects of air traffic control of drones. The scope of activities in this sense is constantly increasing and includes a large number of organizations, agencies and other representative bodies, and therefore "University of Applied Sciences ATM Integration Activity" was established, with the aim of developing the policy of manaing air traffic of military and civil drones. With the final aim of safe integration of drones in the controlled airspace, EUROCONTROL is developing the requirements for drones in ATM environment, which will consist of a set of interoperable criteria for operative authorizations and certification. These requirements assume that the drones will completely adapt in the existing ATM system, rather than the system being adapted in any way for the drones (EUROCONTROL, 2012).

Through its engagement in the integration of pilotless system of air traffic control, EUROCONTROL, follows several principles, and so the operations which include participation of drones must not in any way increase the risk for other participants in air traffic, the ATM procedures for drones shall completely resemble those that are valid for aircraft with human crew, and the insurance of air traffic control services towards drones have to be transparent among airspace controllers at all levels (EUROCONTROL, 2012).

U-Space concept (shown in Table 1) is developed by Single European Sky ATM 
Research Joint Undertaking (SJU), but EUROCONTROL has a crucial role in that concept. U-space is a set of new services relying on a high level of digitalisation and automation of functions and specific procedures designed to support safe, efficient and secure access to airspace for large numbers of drones. U-Space is based on three principles: U-Space should be safe (safety at low altitude levels will be just as good as that for traditional manned aviation), automated (the system will provide information for highly automated or autonomous drones to fly safely and avoid obstacles or collisions) and ready for operations by 2019 - at least for the basic services like registration, e-identification and geo-fencing. The idea is to develop a traffic management system for UAS with functions quite similar to those already existing for manned aviation ATM, but with infrastructures and services adapted to the specificities of drone operations. The key challenge for drone operations is to establish trust with the manned aviation stakeholders who are often still in resistance mode when it comes to sharing air-space and the EUROCONTROL is crucial organization that can help overlapping that gap.

\section{Table 1}

\begin{tabular}{|c|c|c|}
\hline Timescale & UTM services & RPAS integration \\
\hline 2019 plus & 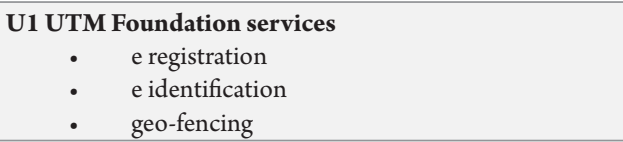 & RPAS 1 - IFR in classes A-C \\
\hline 2022 plus & $\begin{array}{ll}\text { U2 UTM Initial services } \\
\text { - } & \text { flight planning } \\
\text { - } & \text { flight approval } \\
\text { - } & \text { tracking } \\
\text { - } & \text { airspace dynamic information } \\
\text { - } & \text { procedural interface with ATC }\end{array}$ & RPAS 2 - IFR in classes A-G \\
\hline 2027 plus & $\begin{array}{l}\text { U3 UTM Advanced/enhanced services } \\
\begin{array}{l}\text { - capacity management } \\
\text { - } \quad \text { assistance for conflict detection }\end{array}\end{array}$ & \\
\hline 2030 plus & & $\begin{array}{l}\text { RPAS } 3 \text { - IFR and VFR in classes } \\
\text { A-G }\end{array}$ \\
\hline 2035 plus & $\begin{array}{l}\text { U4 UTM Full services } \\
\begin{array}{l}\text { additional new services and integrated } \\
\text { interfaces with manned aviation }\end{array}\end{array}$ & \\
\hline
\end{tabular}

Source: (Syway magazine, 2018)

\subsection{JARUS Approach}

The Joint Authorities for Rulemaking on Unmanned Systems is composed of a group of experts from national aviation authorities (NAAs) and regional aviation safety organisations (JARUS, 2017). Aim is to develop harmonised rules for drones, by recommending technical, safety and operational requirements for the certification of drones and their safe integration into airspace and at aerodromes. JARUS has developed several documents for the development of a EU Regulations for drones such as on the initial operationcentric approach for categories, and is developing the SORA (Specific Operations Risk Assessment) for the specific category. 
The JARUS guidance material aims to facilitate each authority to write their own requirements and to avoid duplicate efforts.

\section{Assessment of Drone Operations Integration in the ATM Environment}

Operational concept of management of drones in the controlled airspace must take in the account the characteristics of drones and current international and national regulatory framework. The principles of integrating drones in the ATM environment should ensure that safety of air navigation system is considered as the most crucial factor, that drones should be equipped with the communication, navigation and surveillance requirements of the airspace within which they operate and that risk-based approach is applied. One of the challenges that Air Navigation Services Provides have to face is that drone represents a broad term. It can be applied to many types of remotely piloted aircraft or systems, with different equipment levels and capabilities.

Operations of drones should be in compliance with the most of the requirements coming out of the ICAO Annex 2 (Rules of the Air).
In addition, drone operations should not make any difference to the daily operations of other airspace users (commercial and general aviation) except when defining special flight procedures. In relation to Annex 2, drone has to have designated pilot-in-command at all times and has to comply with the ATC instructions and clearances. ICAO Annex 11 (Air Traffic Services) prescribes the requirements for the classification of airspace into classes from A to G. It is in the jurisdiction of individual countries to select the classes that correspond to the operative requirements for the provision of air traffic services and for the performance of flights in their airspace. The integration of drones in the operational ATM environment depends on the level of interaction required between the ATC and drone operator and is dependent on the location of the operation and the equipment levels and capability of the drone itself. The term of "isolated operations" refers to operation where direct interaction with ATC is not required and ATC works autonomously around the location where drone is operating. The following figure shows the UAV isolated operations in the ATM environment.

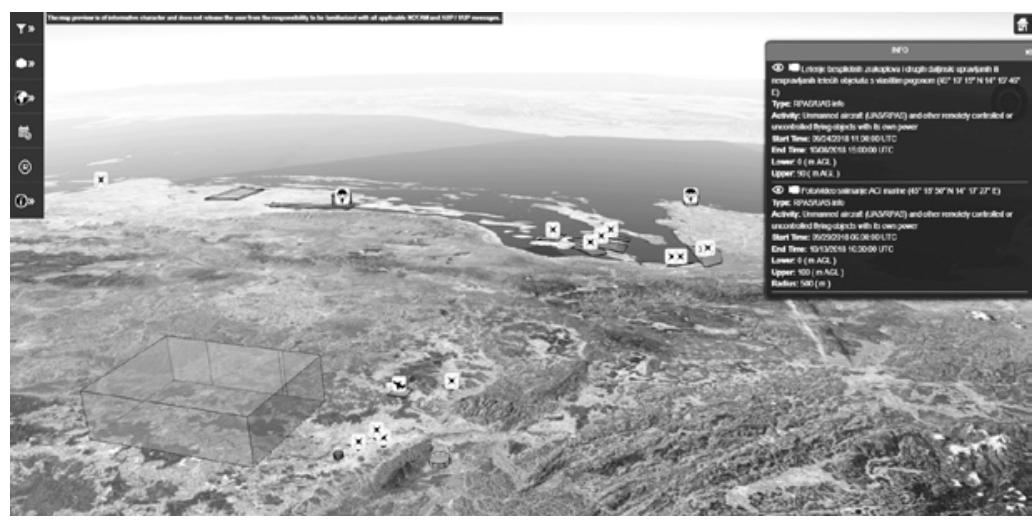

Fig. 2.

Isolated UAV Operations

Source: (Airspace Management Cell, 2018) 
"Synchronised operations" understands the ATC interaction with a drone operator based on the assessment of the characteristics of the location and equipment levels and drone capability. "Integrated operations" refer to drone full application of all operational requirements already applied on conventionally piloted aircraft that can be largely managed through pre-existent systems and processes. Drone operator should inform the ATC on the use of controlled airspace. The initial assessment of drone application in order to facilitate access into the controlled airspace should examine the following factors: location and height of requested operation, the availability of terrain, drone equipment and capability, and any other relevant information. Once the initial assessment has been performed, ATC unit interacting with the live traffic should examine the outcome of the initial assessment, the risk associated with the current drone operation, the compatibility of drone operation with other current and planned operations and the impact of drone operations on overall operations.
When setting up the baseline for the integration of drones into the regulated ATM environment it would be necessary to perform risk-based assessment. The analysis of drone related occurrences for the period 2010-2016 (Fig. 3.), extracted from the European Central Repository (ECR), identified 2141 occurrences of all severity levels. There were 38 occurrences, the majority of which resulted from the drone crash due to technical reasons or loss of control. These occurrences have been classified as accidents, none of the accidents involved fatalities, and there were only 4 minor injuries inflicted to people on the ground. Accidents occur mainly when the drone crashes and is severely damaged due to loss of control, mechanical failure, controlled flight into terrain (CFIT), or midair collision with another aircraft. The most common serious incidents shown on the following figure, are near mid-air collisions. This incident type includes also loss of control and loss of data link cases.

MAC: Airprox/ACAS Alert/loss of..

NAV: Navigation error

UNK: Unknown or undetermined

LOC-I: Loss of control - inflight

ATM: ATM/CNS

SCF-NP: System/component failure or.

SEC: Security related

SCF-PP: Powerplant failure or malfunction

ADRM: Aerodrome

LALT: Low altitude operations

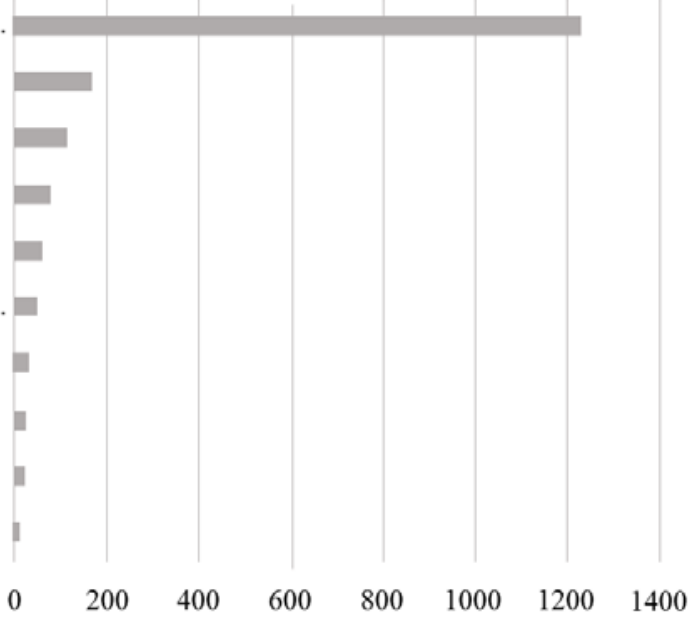

Fig. 3.

Categories of the Reported Drone Occurrences (2010-2016)

Source: (EASA, 2016) 
When performing risk-based assessment of potential situation as loss of control the main identified risks, such as: loss of position data, loss of propulsion, loss of electrical energy, external conditions, crew error and loss of C2 link should be examined. All abovementioned risks could lead to the loss of control situation with a hazard of drone having a ground impact or separation conflict with other traffic. Application of risk-based approach provides the overlook of all possible issues and thus provides the opportunity to cover the drone integration in the ATM environment holistically.

One of the issues of the integration of drones into controlled airspace is the radio communication discipline, as some of the classes have two-way communication requirement. If there is a lack of operator aviation/airspace knowledge, then this issue could introduce a variety of additional integration problems. There is also a difficulty in distinguishing piloting capabilities if there is no recognized training standard or national licensing requirement. According to Chicago Convention, the pilot of every aircraft and other involved members shall be provided with certificates of competency and licenses. Even though the remote pilots and other members of the remote crew are not subject to Article 32 of the Chicago Convention, there is a need to establish a necessary regulatory baseline for the entire community not just for ATC and commercial aviation.

During drone operations, the ground control station is stationary in relation to the ATM network, whereas unmanned aircraft, regardless of the level of autonomy is critically dependent on the input commands by the ground operator. The most significant difference between the operations of drones and conventional aircraft with human crew is reflected in the geographic relation between the ground control station and the ATM operative unit. When operating with drone beyond visual line of sight, pilots are communicating via command and control C2 link. Data links convey vital information to and from drone and ground control station, wirelessly in most cases. This information or data are used for controlling drones manually or through automation by manipulating control surfaces and throttle. The command and control functionality of drones allows operators to know what is happening on board the aircraft while flying. The command and control systems are essential to the operation of any drone. Through C2 subsystem, manual control and autonomous flight is achieved and telemetry exchanged between the aircraft and ground station enabling the monitoring of system health and aircraft flight performance. Through the command and control link, an operator may update aircraft systems and upgrade flight plan. The relationship between a conventional aircraft with human crew and the ATM operative unit is relative, since the communication takes place directly between the authorized ATM unit and the aircraft itself by means of adequate communication channels for data and audio transfer (ICAO, 2011).

The main issue for air traffic controllers concerning the communication with the drone pilot is delay in C2 link or total loss of link connection. The issue with communication delay in $\mathrm{C} 2$ link is that drone response is slower than typical manned aircraft, and due to that, it increases the complexity of operations within the controlled airspace sector and controller's workload. Because there is a possibility to have a total loss of C2 link, 
there is a big concern who and when shall provide clearances to the drone if there is no possibility to communicate with it. In situations like this, controller's workload would increase, as they would have to separate all other aircraft from the predefined drone flight path. This situation is linked with the situational awareness and predictability issues that are becoming more and more complex with introducing drones in the ATM environment. In addition, drones operating in controlled environment should provide collision avoidance at all times and safe separation when there is no positive ATC separation (see and avoid). In order to ensure this requirement, drones should be equipped with Mode C/S transponders, or other approved systems, compatible and cooperative with airborne collision avoidance systems installed on manned aircraft.

Since the current ATM is still not completely a network system, the communication transfer is difficult in case when the unmanned aerial vehicles fly between several different sectors that belong to different air traffic control authorities. Communication transfer that are significant for the aircraft navigation services need to meet the requirements applicable for a certain airspace or operation, in compliance with the decisions of proper aviation authorities. In order to minimize the possibility of external interference, it is necessary to establish, as in the case of aircraft with human crew, special allocation of frequency bands.

Introduction of drones in the controlled airspace could in the future affect the separation standards currently set in the ATM environment. Due to the fact that with the loss of $\mathrm{C} 2$ link and with current separation standards, there is a possibility for creating potential conflict situation between drones and other known traffic. This situation could have even higher impact in terminal zones and around airports, where separation minima is already reduced.

When considering the possibility of performing operations with drones in ATM environment, it should be determined how much these systems are technologically compatible. During their operations, drones require an extremely wide communication channel, which at the same time provides control commands in two-way communication towards the aircraft itself, as well as two-way communication between the aircraft and the air traffic control, i.e. between the air traffic control and the ground control station, which leads to more complex operational requirements during the flight of the UAVs between two or several sector authorities (ICAO, 2011). Figure 4 shows the communication connections in ATM environment between the system user and the air traffic control. 


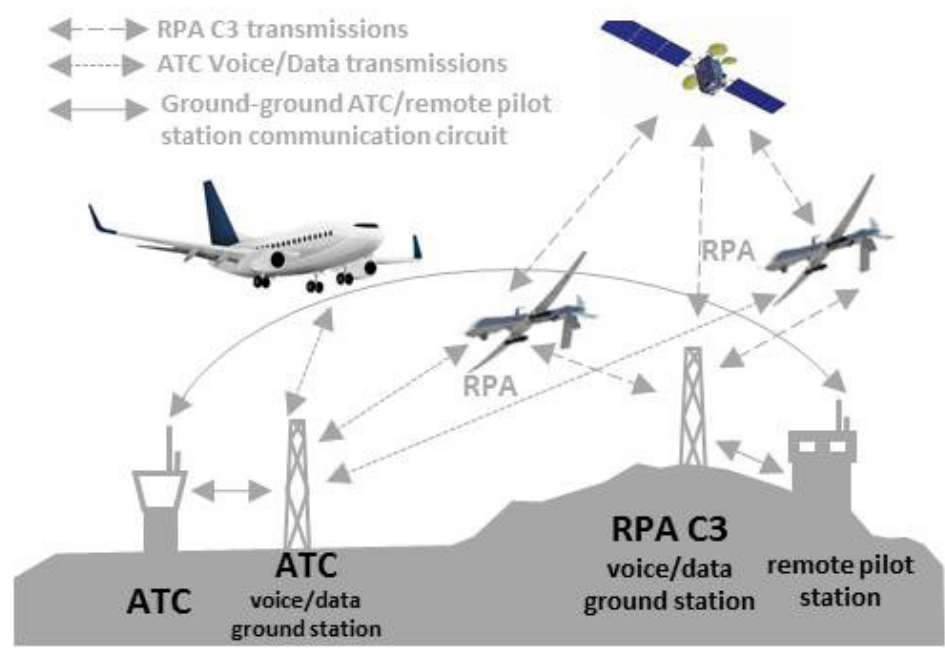

Fig. 4.

Communication Connections in ATM Environment

Source: (ICAO, 2011)

A comprehensive modernization is planned in the air traffic control system so that the system will be more oriented to the network system of information exchange, since the current configuration represents a complex set of independent system interconnected by different technologies by means of geographically dispersed installations. It would be appropriate, as there is a great number of different drone types and their potential usage to develop information booklet for controllers. This information could be based on the specific part of the airspace (i.e. sectors), which would contain all needed information on drone missions, contingency plan in case of C2 link delay or total loss of communication, drone specifications and other needed information.

The growth of capacities in terms of introducing the drones in the ATM environment has to be assured by introducing a proper training for controllers, concerning the drone operations in their local environment. Controllers training concerning the drone operations are handled locally within the in-house training development plans. Training plans currently include several types of drones and possible missions, but in the future as with the evolution of drone market and their potential usage, it should be updated accordingly. Training plans should contain also training on possible risk mitigation measures concerning the loss of C2 link or delay in communication and contingency procedures.

Drones need to be integrated smoothly and introduced fully through the ATM network system with which they have to be equally interoperable. Although these requirements on the one hand represent a challenge in the technological adaptation of drone operations in the ATM environment, on the other hand they represent an opportunity to adapt the development of unmanned systems to the network information exchange. Such manner 
of operation allows faster distribution of critical information within the operations environment, so that the response time of the control loop in case of contingencies during the flight is reduced. Such a structure allows for an even higher autonomy for flying, since it allows the existence of input data even in case of failure of communication between the drone and ground control station. Gradual transformation of the today's ATM system towards future, network-oriented organization, will facilitate to a certain extent and direct the next steps in the development of drones towards full integration in the controlled airspace (ICAO, 2011).

\section{Conclusion}

Introduction of drones into the ATM controlled airspace brings up the everlasting discussion on such diverse topics as licensing and medical qualification of remote pilots, technologies for detect and avoid systems, frequency spectrum, separation standards from other aircraft and development of a robust regulatory framework.

As long as there is no harmonised approach on the EU level there will be no full integration of drones in the European airspace. This conclusion can be drawn up from different regulatory approaches in European countries and especially because drones raise concerns about safety and privacy. It has to be emphasized that public acceptance presents a prerequisite for a successful implementation of drones in the regulated ATM environment. One significant incident or accident between drone and commercial air transport might undermine all the effort undertaken until now.
At this moment, it is not possible to perform the all drone operations in controlled airspace in full compliance with safety standards, since the standards and norms required by such an environment have not been unambiguously determined yet. The highest attention is focused on airworthiness of drones, training and licensing of operators, handling airports, ground control stations for the control and performance of missions and communications and connections between aircraft, operators and air traffic control.

It will be only when the drones are used operatively in controlled airspace that the safety question will be answered whether the requirements and international regulations prescribed by the organizations such as ICAO, EASA and EUROCONTROL can be efficiently performed.

It should be determined as well whether the drones have the sufficient level of autonomy in order to be able to join smoothly in the controlled airspace. The technological environment of the contemporary air traffic, i.e. the results of research and development of different drone types and operations and air traffic control network, indicates that the necessary level has already been achieved, so that there are no barriers for the drones leaving the isolated and entering the controlled airspace. In addition, it needs to bear in mind that as with the higher levels of autonomy the influence of human error is reduced, the possible technology error might still be higher. Even though the human factors are widely elaborated in aviation environment, the levels of automation used in drone industry should still be examined. 
Before drones enter the system of air traffic control, a number of rules, norms and legalregulatory requirements need to be met. Therefore, licensing of airworthiness and training of the crews are not sufficient as such. There should be interaction between the crew managing the drone flight in the controlled airspace and the adequate flight control unit. At every moment, a person should be delegated who is responsible for the movement of the drone, such as the pilot responsible for an aircraft, since in conventional aviation the pilot carries the largest responsibility for all the aspects of flying that are not under direct jurisdiction of the air traffic control. In order to mitigate the possible drone integration issues identified, the best proposed approach would be to introduce the risk-based model. This model would enable identification of all risks that could encounter before and during the drone integration in the controlled airspace and it would as an output define all mitigation measures for the risks identified. Risk-based approach would also allow all stakeholders to get the holistic approach when developing a "business case" for introduction of drones in the controlled airspace.

Even though this paper has identified the current main potential issues in the drone integration process, the main question that should be: "Until when shall the ATM environment influence the drone operations or it might be that in the future the situation would go other way around?" Meaning that even greater level of introduction of drones in the commercial domains and controlled ATM environment, and the technology development with certain levels of automatization to be reached, shall influence the existing regulatory framework and ask for significant modifications of rules and procedures.

\section{Acknowledgements}

This paper was made as a part of the bilateral Croatian-Chinese research project Development of Low-Altitude Airspace Surveillance System Based on the UAV Platform.

\section{References}

Airspace Management Cell (AMC). 2018. Current Situation in the Air. Available from internet: <https:// amc.crocontrol.hr/Current-situation-anonymous-users $>$.

EASA. 2015. Concept of Operations for Drones: A risk based approach to regulation of unmanned aircraft. Cologne, Germany.

EASA. 2016. UAS Safety Risk Portfolio and Analysis. Cologne, Germany.

EC. 2015. Riga Declaration on Remotely Piloted Aircraft (drones). Framing the future of the aviation. 6 March 2015, Riga, Latvia.

Embedded Vision Summit. 2016. Vision Processing Opportunities in Drones. May 2016, Santa Clara, USA.

ERSG. 2013. Roadmap for the integration of civil RemotelyPiloted Aircraft Systems into the European Aviation System. Final report from the European RPAS Steering Group, Brussels, Belgium, June 2013, 15 p.

EUROCONTROL. 2012. EUROCONTROL Specifications for the Use of Military Unmanned Aerial Vehicles as Operational Air Traffic Outside Segregated Airspace. Brussels, Belgium.

EUROCONTROL. 2017. RPAS workshop addressing the current ATM operational integration issues, 2017, Brussels, Belgium.

ICAO. 2011. Unmanned Aircraft Systems (UAS). ICAO Circular 328-AN/190, ISBN 978-92-9231-751-5, Montreal, Canada. 
ICAO. 2015. Manual on Remotely Piloted Aircraft Systems (RPAS). First Edition - 2015, ISBN 978-92-9249 718-7, Montreal, Canada.

JARUS. 2017. JARUS - Who We Are \& What We Do. Joint Authorities for Rulemaking on Unmanned Systems. Available from Internet: <http://jarus-rpas.org/whowe-are $>$.
Syway magazine. 2018. EUROCONTROL sits at the heart of regulatory, research and operational drone integration work. EUROCONTROL, Brussels, Belgium, 25 p.

Teal Group Corporation. 2014. World Unmanned Aerial Vehicle Systems. 2014 Market Profile and Forecast, 2014 Edition. Teal Group Corporation, Fairfax, USA, 455 p. 